\title{
INITIAL AND RELATIVE LIMITING BEHAVIOUR OF TEMPERATURES ON A STRIP
}

\author{
N. A. WATSON
}

(Received 16 June 1981)

Communicated by R. Vyborny

\begin{abstract}
Let $u$ be a solution of the heat equation which can be written as the difference of two non-negative solutions, and let $v$ be a non-negative solution. A study is made of the behaviour of $u(x, t) / v(x, t)$ as $t \rightarrow 0+$. The methods are based on the Gauss-Weierstrass integral representation of solutions on $\left.R^{n} \times\right] 0, a[$ and results on the relative differentiation of measures, which are employed in a novel way to obtain several domination, non-negativity, uniqueness and representation theorems.
\end{abstract}

1980 Mathematics subject classification (Amer. Math. Soc.): 35 K 05, 35 B 05, 35 C 15, 28 A 15.

Let $W$ denote the Gauss-Weierstrass kernel, defined, for all $\left.(x, t) \in \mathbf{R}^{n} \times\right] 0, \infty[$, by $W(x, t)=(4 \pi t)^{-n / 2} \exp \left(-\|x\|^{2} / 4 t\right)$, and let $\mu$ be a locally finite, signed Borel measure on $\mathbf{R}^{n}$. Then $u$, given by the convolution

$$
u(x, t)=\int_{\mathbf{R}^{n}} W(x-y, t) d \mu(y),
$$

is called the Gauss-Weierstrass integral of $\mu$, provided that the integral exists. If the integral exists and is finite at a point $\left(x_{0}, t_{0}\right)$,then $u$ is a temperature, that is, a solution of the heat equation, on $\left.\mathbf{R}^{n} \times\right] 0, t_{0}[$. Conversely, if $v$ is a temperature on a strip $\left.\mathbf{R}^{n} \times\right] 0, c$, or on a half-space $\left.\mathbf{R}^{n} \times\right] 0, \infty$ [, and $v$ can be written as the difference of two non-negative temperatures, then $v$ has a representation as the Gauss-Weierstrass integral of some signed measure $\nu$. For details and references, see [14]. We write $u=W \mu$ if $u$ and $\mu$ are related by (1), and always assume that such integrals are finite on some strip or half-space $\left.\mathbf{R}^{n} \times\right] 0, c[$, where $0<c \leqslant \infty$.

In [5, Theorem 5.2], Doob proved that, if $u=W \mu$ and $v=W \nu$, then

$$
\lim _{t \rightarrow 0} \frac{u(x, t)}{v(x, t)}
$$

(c) Copyright Australian Mathematical Society 1982 
exists a.e. [| $\nu \mid]$ on $\mathbf{R}^{n}$, and is then equal to the Radon-Nikodym derivative of $\mu$ with respect to $\nu$. Similar results have been proved for harmonic functions, and in more general situations with different limits (see [3] for references), but further study of the behaviour of $u / v$, and application of the results about $u / v$, have apparently been neglected. In [3], Brelot mentioned one simple application of an analogous result for harmonic functions. In [19], new results and applications were given for Gauss-Weierstrass integrals, and the present paper contains further theorems, but generally of a different nature. We use the following basic result [19, Theorem 1].

Let $u=W \mu$ and $v=W \nu$, where $\nu$ is non-negative, and let $x \in \mathbf{R}^{n}$. If $\nu(B(x, r))$ $>0$ for all closed balls $B(x, r)$ in $\mathbf{R}^{n}$ with positive radius $r$, then

$$
\begin{aligned}
\liminf _{r \rightarrow 0} \frac{\mu(B(x, r))}{\nu(B(x, r))} & \leqslant \liminf _{t \rightarrow 0} \frac{u(x, t)}{v(x, t)} \leqslant \limsup _{t \rightarrow 0} \frac{u(x, t)}{v(x, t)} \\
& \leqslant \limsup _{r \rightarrow 0} \frac{\mu(B(x, r))}{\nu(B(x, r))}
\end{aligned}
$$

The first theorem of the present paper is concerned with the upper and lower limits of the quotient $\mu(B(x, r)) / \nu(B(x, r))$ as $r \rightarrow 0$ and, in view of the above result, it has immediate application to the relative behaviour of temperatures. We are thus able to prove some new domination, non-negativity, uniqueness and representation theorems for temperatures. These results include a multi-variable version of a theorem of Gehring [6, Theorem 10], analogues of results for harmonic functions on a disc in the plane due to Bruckner, Lohwater and Ryan [4, Theorems 2 and 3], Hall [8, Theorem 4], and Lohwater [12], and a much more general version of a recent improvement for temperatures [15, Theorem 5] of a result of Krzyżański [11, Theorem 5].

In addition, we are able to compare the strengths of singularities of GaussWeierstrass integrals of singular and absolutely continuous measures. For example, it is well-known that, if $\mu(\{x\})=\lambda \neq 0$ and $u=W \mu$, then $u(x, t) \sim$ $(4 \pi t)^{-n / 2} \lambda$ as $t \rightarrow 0$, whereas if $\mu(\{x\})=0$ then $u(x, t)=o\left(t^{-n / 2}\right)$ as $t \rightarrow 0$. We shall show that, if $\nu$ is non-negative and absolutely continuous, $v=W \nu, \mu$ is non-negative and concentrated on the set where $v(x, t)$ is unbounded as $t \rightarrow 0$, and $u=W \mu$, then $v(x, t)=o(u(x, t))$ as $t \rightarrow 0$ for $\mu$-almost every point $x$ in $\mathbf{R}^{n}$.

We also give two theorems which show that we can sometimes deduce from the behaviour of $u / v$ that $\mu$ or $\nu$ must be concentrated on some particular set.

Given $x=\left(x_{1}, \ldots, x_{n}\right) \in \mathbf{R}^{n}$ and $r>0$, we put $\|x\|=\left(x_{1}^{2}+\cdots+x_{n}^{2}\right)^{1 / 2}$ and $B(x, r)=\left\{y \in \mathbf{R}^{n}:\|x-y\| \leqslant r\right\}$. Every measure in this paper is a locally finite, signed Borel measure on $\mathbf{R}^{n}$. The letter $m$ is used to denote Lebesgue measure on 
$\mathbf{R}^{n}$. We shall call a measure $\nu$ strictly positive if $\nu(B(x, r))>0$ for all $x \in \mathbf{R}^{n}$ and $r>0$. The positive, negative and total variations of a measure $\mu$ are denoted by $\mu^{+}, \mu^{-}$and $|\mu|$.

The following temperature occurs in several of our theorems. Given a number $\kappa \geqslant 0$, we let $V_{\kappa}$ denote the Gauss-Weierstrass integral of the function $x \mapsto$ $\exp \left(\kappa\|x\|^{2}\right)$, that is,

$$
V_{\kappa}(x, t)=(1-4 \kappa t)^{-n / 2} \exp \left\{\kappa\|x\|^{2} /(1-4 \kappa t)\right\}
$$

for all $(x, t)$ in $\left.\mathbf{R}^{n} \times\right] 0,(4 \kappa)^{-1}\left[\right.$ if $\kappa>0$, in $\left.\mathbf{R}^{n} \times\right] 0, \infty[$ if $\kappa=0$. Of course, $V_{0}(x, t)=1$.

Finally, if $u$ is a temperature and $v$ is a non-negative temperature such that $u \leqslant v$ on $\left.\mathbf{R}^{n} \times\right] 0, c[$, then $v$ is called a pasitive thermic majorant of $u$ on $\left.\mathbf{R}^{n} \times\right] 0, c[$. For details and references, see [18].

\section{Relative differentiation of measures}

In this section we present several results on the behaviour of $\mu(B(x, r)) / \nu(B(x, r))$ as $r \rightarrow 0$, which we require later. The lemmas are all due to Besicovitch [1,2], but one new theorem is also given.

LEMMA 1. If $\mu$ and $\nu$ are non-negative measures on $\mathbf{R}^{n}$, then

$$
\lim _{r \rightarrow 0} \frac{\mu(B(x, r))}{\nu(B(x, r))}
$$

exists and is finite for $\nu$-almost all $x$ in $\mathbf{R}^{n}$.

This result is proved, in the case $n=2$, in [1, Theorem 2]. As with all the results in $[1,2]$, the proof carries over to the general case.

Lemma 2. Let $\mu$ and $\nu$ be non-negative measures on $\mathbf{R}^{n}$, and let $Y$ be a Borel set such that $\mu(Y)=0$. Then

$$
\lim _{r \rightarrow 0} \frac{\mu(B(x, r))}{\nu(B(x, r))}=0
$$

for $\nu$-almost all $x$ in $Y$.

See [1, Theorem 3]. 
LEMMA 3. If $\mu$ is a non-negative measure, and if a family $F$ of balls covers a Borel set $E$ in such a way that, for each $x \in E$, there is a ball $B(x, r)$ in $F$ with arbitrarily small $r$, then $F$ contains a subfamily of disjoint balls whose union $H$ has the property that $\mu(E \backslash H)=0$.

This is a special case of [2, Theorem 3].

We now come to a new theorem, which generalizes and strengthens a result which was stated, without proof and for the case $\nu=m$ only, by Rosenbloom in [13].

THEOREM 1. Let $\mu$ and $\nu$ be measures on $\mathbf{R}^{n}, \nu$ being strictly positive. If

$$
\limsup _{r \rightarrow 0} \frac{\mu(B(x, r))}{\nu(B(x, r))}>-\infty
$$

for all $x \in \mathbf{R}^{n}$, and

$$
\limsup _{r \rightarrow 0} \frac{\mu(B(x, r))}{\nu(B(x, r))} \geqslant 0
$$

for $\nu$-almost all $x \in \mathbf{R}^{n}$, then $\mu$ is non-negative.

Proof. For each non-negative integer $k$, let $P_{k}$ denote the set of all $x$ for which

$$
\underset{r \rightarrow 0}{\limsup } \frac{\mu(B(x, r))}{\nu(B(x, r))} \geqslant-k \text {. }
$$

Then (3) implies that

$$
\bigcup_{k=0}^{\infty} P_{k}=\mathbf{R}^{n}
$$

and (4) shows that

$$
\nu\left(\mathbf{R}^{n} \backslash P_{0}\right)=0 .
$$

Let $\varepsilon>0$. To each $x$ in $P_{0}$ there corresponds a positive null sequence $\left\{r_{i}\right\}$ such that

$$
\mu\left(B\left(x, r_{i}\right)\right) \geqslant-\varepsilon \nu\left(B\left(x, r_{i}\right)\right)
$$

for all $i$. For each $k>0$ we have $\nu\left(P_{k} \backslash P_{k-1}\right)=0$, by (6), so that there is an open set $V_{k} \supseteq P_{\mathrm{k}} \backslash \mathrm{P}_{k-1}$ such that

$$
\nu\left(V_{k}\right)<2^{-k} \varepsilon
$$

To each $x \in P_{k} \backslash P_{k-1}$ there corresponds a positive null sequence $\left\{r_{i}\right\}$ such that

$$
B\left(x, r_{i}\right) \subseteq V_{k} \text { and } \mu\left(B\left(x, r_{i}\right)\right) \geqslant-(k+\varepsilon) \nu\left(B\left(x, r_{i}\right)\right)
$$

for all $i$. 
Let $E$ be any bounded open set in $\mathbf{R}^{n}$. Consider the family $F$ of all balls $B\left(x, r_{i}\right) \subseteq E$ such that either $x \in E \cap P_{0}$ and (7) holds, or $x \in E \cap\left(P_{k} \backslash P_{k-1}\right)$ and (8) holds. In view of (5) the family $F$ covers $E$, and for each $x \in E$ there is a ball $B(x, r)$ in $F$ with arbitrarily small $r$. Therefore, by Lemma 3, there is a sequence $\left\{C_{j}\right\}$ of disjoint members of $F$ such that

$$
|\mu|\left(E \backslash\left(\bigcup_{j} C_{j}\right)\right)=0 .
$$

For each $k \geqslant 0$, let $\left\{\Gamma_{k j}\right\}$ denote the (possibly finite or empty) subsequence consisting of those $C_{j}$ whose centres lie in $P_{k} \backslash P_{k-1}$ if $k>0$, in $P_{0}$ if $k=0$. Then

$$
\begin{aligned}
\mu(E) & =\mu\left(\bigcup_{j} C_{j}\right)=\sum_{k=0}^{\infty}\left(\sum_{j} \mu\left(\Gamma_{k j}\right)\right) \\
& \geqslant-\sum_{k=0}^{\infty}(k+\varepsilon)\left(\sum_{j} \nu\left(\Gamma_{k j}\right)\right) \\
& \geqslant-\varepsilon \nu(E)-\sum_{k=1}^{\infty}(k+\varepsilon) \nu\left(V_{k}\right) \\
& \geqslant-\varepsilon\left(\nu(E)+\sum_{k=1}^{\infty} 2^{-k}(k+\varepsilon)\right) .
\end{aligned}
$$

Since $\varepsilon$ is arbitrary, it follows that $\mu(E) \geqslant 0$.

Therefore $\mu^{+}(E) \geqslant \mu^{-}(E)$ for all bounded open sets $E$. Using the regularity properties of $\mu^{+}$and $\mu^{-}$, we deduce that $\mu^{+}(S) \geqslant \mu^{-}(S)$ for every $\mu$-measurable set $S$. This proves the theorem.

COROLlaRY. Let $\mu$ and $\nu$ be measures on $\mathbf{R}^{n}$ such that $\nu$ is strictly positive. If

$$
\lim _{r \rightarrow 0} \frac{\mu(B(x, r))}{\nu(B(x, r))}
$$

is finite whenever it exists, and is zero $\nu$-almost everywhere, then $\mu=0$.

Proof. By Lemma 1, the limit in (9) exists and is finite $\nu$-almost everywhere. Therefore the hypotheses of Theorem 1 are satisfied with $\mu$ itself, and also with $\mu$ replaced by $-\mu$ throughout. Hence both $\mu$ and $-\mu$ are non-negative, and the corollary is proved. 


\section{Some applications of Besicovitch's results}

The results presented here are all consequences of the above lemmas and the fundamental inequalities in (2).

THEOREM 2. Let $\mu$ and $\nu$ be non-negative measures on $\mathbf{R}^{n}$, and let $Y$ be a Borel set such that $\mu(Y)=0$. If $u=W \mu$ and $v=W \nu$ on $\left.\mathbf{R}^{n} \times\right] 0, c[$, then

$$
u(x, t)=o(v(x, t)) \text { as } t \rightarrow 0
$$

for $\nu$-almost all $x \in Y$. In particular, if $\mu$ and $\nu$ are mutually singular, then (10) holds for $\boldsymbol{\nu}$-almost every $x \in \mathbf{R}^{n}$.

Proof. By (2) and Lemma 2, we have

$$
\lim _{t \rightarrow 0} \frac{u(x, t)}{v(x, t)}=\lim _{r \rightarrow 0} \frac{\mu(B(x, r))}{\nu(B(x, r))}=0
$$

for $\nu$-almost all $x \in Y$. This proves the first part, and the second now follows by taking $Y$ to be any Borel set such that $\mu(Y)=0$ and $\nu\left(\mathbf{R}^{n} \backslash Y\right)=0$.

We now use Theorem 2 to show that the initial singularities of $W \mu$, where $\mu$ is absolutely continuous with respect to $m$, are milder than those of a corresponding $W \nu$ with $\nu$ singular with respect to $m$, at least $\nu$-a.e.

THEOREM 3. Let $u=W \mu$, where $\mu$ is non-negative and absolutely continuous with respect to $m$, and put

$$
Z=\left\{x: \limsup _{t \rightarrow 0} u(x, t)=\infty\right\} .
$$

If $\nu$ is a non-negative measure concentrated on $Z$, and $v=W \nu$, then

$$
u(x, t)=o(v(x, t)) \text { as } t \rightarrow 0
$$

for $\nu$-almost every $x \in \mathbf{R}^{n}$.

Proof. Since $u(x, t)$ tends to a finite limit as $t \rightarrow 0$ for $m$-almost every $x$ in $\mathbf{R}^{n}$, we see that $m(Z)=0$ and hence that $\mu(Z)=0$. Since $\nu$ is concentrated on $Z$, we deduce that $\mu$ and $\nu$ are mutually singular, and the result now follows from Theorem 2.

The next theorem is analogous to certain results of Brelot [3] on various limits of quotients of positive harmonic or superharmonic functions. 
THEOREM 4. Let $u=W \mu$ and $v=W \nu$, where $\nu$ is non-negative on $\mathbf{R}^{n}$. The limit

$$
\lim _{t \rightarrow 0} \frac{v(x, t)}{u(x, t)}
$$

exists and is non-zero $\nu$-a.e. In particular

$$
\lim _{t \rightarrow 0} v(x, t)
$$

exists and is strictly positive $\nu$-a.e.

Proof. Let $N=\{x: \nu(B(x, r))=0$ for some $r>0\}$. Then $N$ is an open set and $\nu(N)=0$. Since the inequalities in (2) are applicable to any $x$ in $\mathbf{R}^{n} \backslash N$, it follows from (2) and Lemma 1 that

$$
\lim _{t \rightarrow 0} \frac{u(x, t)}{v(x, t)}=\lim _{r \rightarrow 0} \frac{\mu(B(x, r))}{\nu(B(x, r))}
$$

exists and is finite for $\nu$-almost all $x$ in $\mathbf{R}^{n}$. Hence the limit in (11) exists and is non-zero $\nu$-a.e. in $\mathbf{R}^{n}$. The second part of the theorem follows from the first by taking $u=1$.

Corollary. Let $u=W \mu$ and $v=W \nu$, where $\nu$ is non-negative on $\mathbf{R}^{n}$. The set of $x$ for which

$$
\liminf _{t \rightarrow 0} \frac{v(x, t)}{u(x, t)}=0
$$

has $\nu$-measure zero. In particular,

$$
\nu\left(\left\{x: \liminf _{t \rightarrow 0} v(x, t)=0\right\}\right)=0 .
$$

Our final result in this section is a generalization of [15, Corollary, page 278], which corresponds to the case where $\nu=m$ and $S=\varnothing$. In view of Theorem 2, it is essentially a sharpened form of the above Corollary for the case where $\mu$ and $\nu$ are mutually singular.

THEOREM 5. Let $u=W \mu$ and $v=W \nu$, where $\mu$ is non-negative and $\nu$ is strictly positive, and put

$$
E=\{x: u(x, t) / v(x, t) \text { tends to a finite limit as } t \rightarrow 0\}
$$

and

$$
S=\{x: u(x, t) / v(x, t) \text { tends to } \infty \text { as } t \rightarrow 0\} .
$$

If $u(x, t)=o(v(x, t))$ as $t \rightarrow 0$, for $\nu$-almost all $x$ in $E$, then $\mu$ is concentrated on $S$. 
Proof. If $x \notin S$, then either

(i) $u(x, t) / v(x, t)$ tends to zero as $t \rightarrow 0$, or

(ii) $u(x, t) / v(x, t)$ tends to a finite, non-zero limit as $t \rightarrow 0$, or

(iii) $u(x, t) / v(x, t)$ tends neither to a limit nor to infinity.

Let $A, B$ and $C$ denote the sets where (i), (ii) and (iii) hold respectively. By the Corollary to Theorem $4, \mu(A)=0$. By hypothesis, $\nu(B)=0$. By [5, Theorem 5.2], $\nu\left(\mathbf{R}^{n} \backslash E\right)=0$ and hence $\nu(C)=0$. Therefore $\nu(B \cup C)=0$, and hence Lemma 2 implies that

$$
\lim _{r \rightarrow 0} \frac{\nu(B(x, r))}{\mu(B(x, r))}=0
$$

for $\mu$-almost all $x \in B \cup C$. The inequalities in (2) now show that

$$
\lim _{t \rightarrow 0} \frac{u(x, t)}{v(x, t)}=\lim _{r \rightarrow 0} \frac{\mu(B(x, r))}{\nu(B(x, r))}=\infty
$$

$\mu$-a.e. on $B \cup C$. The definitions of $B$ and $C$ now imply that $\mu(B \cup C)=0$, and hence $\mu\left(\mathbf{R}^{n} \backslash S\right)=0$, as required.

\section{Domination, non-negativity and uniqueness theorems for temperatures}

We now present some immediate consequences of Theorem 1.

THEOREM 6. Let $u=W \mu$ and $v=W \nu$ on $\left.R^{n} \times\right] 0, c[$, where $\nu$ is strictly positive and $0<c \leqslant \infty$. If

$$
\limsup _{t \rightarrow 0} \frac{u(x, t)}{v(x, t)}>-\infty
$$

for all $x \in \mathbf{R}^{n}$, and

$$
\limsup _{t \rightarrow 0} \frac{u(x, t)}{v(x, t)} \geqslant A
$$

for $\nu$-almost every $x \in \mathbf{R}^{n}$, then $u \geqslant A v$ on $\left.R^{n} \times\right] 0, c[$.

Proof. We may suppose that $A=0$, since we could replace $u$ by $u-A v$ throughout. By (2),

$$
\limsup _{t \rightarrow 0} \frac{u(x, t)}{v(x, t)} \leqslant \limsup _{r \rightarrow 0} \frac{\mu(B(x, r))}{\nu(B(x, r))}
$$

for all $x \in \mathbf{R}^{n}$, so that (12) and (13) imply that the hypotheses of Theorem 1 are satisfied. Hence $\mu \geqslant 0$, and therefore $u \geqslant 0$. 
As a consequence of Theorem 6, we can extend a result of Gehring [6, Theorem 10] to the case of an arbitrary $n$, and thus sharpen [18, Theorem 3] and extend [17, Theorem 5] to $\mathbf{R}^{n}$ for all $n$.

THEOREM 7. Let $u=W \mu$ on $\left.\mathbf{R}^{n} \times\right] 0, c[$. If

$$
\lim _{t \rightarrow 0} u(x, t)>-\infty
$$

for all $x$ at which the limit exists, and

$$
\lim _{t \rightarrow 0} u(x, t) \geqslant A
$$

for $m$-almost every $x \in \mathbf{R}^{n}$, then $u \geqslant A$ on $\mathbf{R}^{n} \times 10, c[$.

Proof. Take $\nu=m$ in Theorem 6.

Theorem 6 also gives rise to the following uniqueness result.

TheOREM 8. Let $u=W \mu$ and $v=W \nu$ on $\left.\mathbf{R}^{n} \times\right] 0, c[$, where $\nu$ is strictly positive. If

$$
\liminf _{t \rightarrow 0} \frac{|u(x, t)|}{v(x, t)}<\infty
$$

for all $x \in \mathbf{R}^{n}$, and

$$
\liminf _{t \rightarrow 0} \frac{u(x, t)}{v(x, t)}=0
$$

for $\nu$-almost every $x \in \mathbf{R}^{n}$, then $u=0$ throughout $\left.\mathbf{R}^{n} \times\right] 0, c[$.

Proof. By applying Theorem 6 to $-u$ and $v$, we deduce that $u \leqslant 0$. Hence (14) implies that

$$
\lim _{t \rightarrow 0} \frac{u(x, t)}{v(x, t)}=0
$$

for $\nu$-almost every $x \in \mathbf{R}^{n}$. Another application of Theorem 6 now shows that $u \geqslant 0$, and the result is proved.

If we put $\nu=m$ in Theorem 8, we obtain a strengthened form of a result which was announced, without proof, in [13], and incorrectly demonstrated in [7]. See [15, page 278] for further details. The result is also analogous to one due to Lohwater [12, Corollary] for harmonic functions on a disc in the plane. 
THEOREM 9. Let $u=W \mu$ on $\left.\mathbf{R}^{n} \times\right] 0, c[$. If

$$
\liminf _{t \rightarrow 0}|u(x, t)|<\infty
$$

for all $x \in \mathbf{R}^{n}$, and

$$
\liminf _{t \rightarrow 0} u(x, t)=0
$$

for m-almost every $x \in \mathbf{R}^{n}$, then $u=0$ throughout $\left.\mathbf{R}^{n} \times\right] 0, c[$.

Another interesting consequence of Theorem 6 is motivated by analogy with recent work of Kuran [10]. It implies that condition (15) in Theorem 9 can be weakened in a particular way, without affecting the conclusion of the theorem (cf. the proof of Theorem 8).

We first recall [16, Theorem 11]. If $Z \subseteq \mathbf{R}^{n}$ and $m(Z)=0$, then there exists a positive temperature $v$ on $\left.\mathbf{R}^{n} \times\right] 0, \infty[$ such that $v(x, t) \rightarrow \infty$ as $(x, t) \rightarrow(y, 0)$ for all $y \in Z$. We can obviously suppose that $v \geqslant 1$, since $v+1$ has similar properties.

THEOREM 10. Let $u=W \mu$ on $\left.R^{n} \times\right] 0, c[$, and suppose that

$$
\liminf _{t \rightarrow 0} u(x, t) \leqslant A
$$

for all $x \in \mathbf{R}^{n} \backslash Z$, where $m(Z)=0$. Let $v$ be a temperature such that $v \geqslant 1$ on $\left.\mathbf{R}^{n} \times\right] 0, c[$ and $v(x, t) \rightarrow \infty$ as $t \rightarrow 0$ for all $x \in Z$. If

$$
\liminf _{t \rightarrow 0} \frac{u(x, t)}{v(x, t)} \leqslant 0
$$

for all $x \in Z$, then $u \leqslant A$ on $\left.\mathbf{R}^{n} \times\right] 0, c[$.

Proof. There is a non-negative measure $\nu$ on $\mathbf{R}^{n}$ such that $v=W \nu$ on $\left.\mathbf{R}^{n} \times\right] 0, c$. Since $v \geqslant 1$, we have $\nu \geqslant m$ and hence $\nu$ is strictly positive. We can suppose that $A=0$, since we could replace $u$ by $u-A$ throughout. It follows from (16) and (17) that

$$
\liminf _{t \rightarrow 0} \frac{u(x, t)}{v(x, t)} \leqslant 0
$$

for all $x \in \mathbf{R}^{n}$, so that Theorem 6 gives the desired result.

In the next section we shall use Theorem 10 to prove some new representation theorems for temperatures. 


\section{Representation theorems}

The theorems of this section feature a countable set $C$. We allow this set to be finite or empty, but retain the notation for a countably infinite set.

The first result is analogous to one due to Bruckner, Lohwater and Ryan [4, Theorem 3] for harmonic functions on the unit disc in $\mathbf{R}^{2}$, at least when $A=0$. Another special case, in which $C=\varnothing$, parallels [4, Theorem 2].

THEOREM 11. Let $u=W \mu$ on $\left.\mathbf{R}^{n} \times\right] 0, c\left[\right.$, and let $C=\left\{x_{j}\right\}_{j \geqslant 1}$ be a sequence of points in $\mathbf{R}^{n}$. If there is a real constant $A$, and a non-negative constant $\kappa$, such that

$$
\liminf _{t \rightarrow 0} u(x, t) \leqslant A \exp \left(\kappa\|x\|^{2}\right)
$$

for m-almost all $x \in \mathbf{R}^{n}$, and

$$
\liminf _{t \rightarrow 0} u(x, t)<\infty
$$

for all $x \in \mathbf{R}^{n} \backslash C$, then $u$ can be written in the form

$$
u(x, t)=A V_{\kappa}(x, t)-h(x, t)+\sum_{j=1}^{\infty} \mu^{+}\left(\left\{x_{j}\right\}\right) W\left(x-x_{j}, t\right)
$$

on $\left.\mathbf{R}^{n} \times\right] 0, \min \left\{c,(4 \kappa)^{-1}\right\}\left[\right.$ if $\kappa>0$, on $\mathbf{R}^{n} \times 10, c[$ if $\kappa=0$, where $h$ is a non-negative temperature and $V_{\kappa}$ is as defined in Section 1.

Proof. If we put $u^{*}=u-A V_{\kappa}$, then (18) becomes

$$
\liminf _{t \rightarrow 0} u^{*}(x, t) \leqslant 0
$$

for $m$-almost all $x \in \mathbf{R}^{n}$, and (19) holds with $u^{*}$ in place of $u$. If we prove the result for $u^{*}$, then the result for $u$ will follow immediately. We may therefore suppose that $A=0$ and $\kappa=0$.

Let $\varepsilon>0$. For each $j$, put $\lambda_{j}=\mu^{+}\left(\left\{x_{j}\right\}\right)+\varepsilon 2^{-j}$, and let

$$
w(x, t)=u(x, t)-\sum_{j=1}^{\infty} \lambda_{j} W\left(x-x_{j}, t\right)
$$

for all $\left.(x, t) \in \mathbf{R}^{n} \times\right] 0, c[$. Since

$$
\begin{aligned}
\sum_{j=1}^{\infty} \lambda_{j} W\left(x-x_{j}, t\right) & \leqslant \sum_{j=1}^{\infty} \mu^{+}\left(\left\{x_{j}\right\}\right) W\left(x-x_{j}, t\right)+\varepsilon(4 \pi t)^{-n / 2} \sum_{j=1}^{\infty} 2^{-j} \\
& \leqslant \int_{\mathbf{R}^{n}} W(x-y, t) d \mu^{+}(y)+\varepsilon(4 \pi t)^{-n / 2}<\infty
\end{aligned}
$$

for all $\left.(x, t) \in \mathbf{R}^{n} \times\right] 0, \infty[$, it follows from [14, Lemma 1] that $w$ is a temperature. 
Let $Z$ denote the set of points where (18) fails to hold, so that $m(Z)=0$. Let $v$ be a temperature such that $v \geqslant 1$ on $\left.\mathbf{R}^{n} \times\right] 0, c[$ and $v(x, t) \rightarrow \infty$ as $t \rightarrow 0$ for all $x \in Z$. Since $w \leqslant u$, for all $x \in \mathbf{R}^{n} \backslash Z$ we have

$$
\liminf _{t \rightarrow 0} w(x, t) \leqslant 0 .
$$

Next, for each $j$ let $\delta_{j}$ denote the Dirac $\delta$-measure concentrated at $x_{j}$. Then $w=W \eta$, where $\eta=\mu-\sum_{j=1}^{\infty} \lambda_{j} \delta_{j}$, and for each $j$ we have $\eta\left(\left\{x_{j}\right\}\right)=\mu\left(\left\{x_{j}\right\}\right)-$ $\lambda_{\text {, }}<0$. Therefore $w\left(x_{j}, t\right) \sim \eta\left(\left\{x_{j}\right\}\right)(4 \pi t)^{-n / 2}$ as $t \rightarrow 0$, in view of [19, Examples 1 and 2]. Thus we see that

$$
\lim _{t \rightarrow 0} w(x, t)=-\infty
$$

for all $x \in C$. Finally, if $x \in Z \backslash C$ we have

$$
\liminf _{t \rightarrow 0} w(x, t) \leqslant \liminf _{t \rightarrow 0} u(x, t)<\infty
$$

by (19), so that

$$
\liminf _{t \rightarrow 0} \frac{w(x, t)}{v(x, t)} \leqslant 0 .
$$

It follows from (21), (22), (23) and Theorem 10 that $w \leqslant 0$ on $\left.\mathbf{R}^{n} \times\right] 0, c[$.

Therefore, in view of (20),

$$
u(x, t) \leqslant \sum_{j=1}^{\infty} \mu^{+}\left(\left\{x_{j}\right\}\right) W\left(x-x_{j}, t\right)+\varepsilon(4 \pi t)^{-n / 2}
$$

for all $\left.(x, t) \in \mathbf{R}^{n} \times\right] 0, c$ and all $\varepsilon>0$. Making $\varepsilon \rightarrow 0$, we obtain

$$
u(x, t) \leqslant \sum_{j=1}^{\infty} \mu^{+}\left(\left\{x_{j}\right\}\right) W\left(x-x_{j}, t\right) .
$$

The sum on the right is therefore a positive thermic majorant of $u$ on $\left.\mathbf{R}^{n} \times\right] 0, c[$, and hence majorizes the least such majorant. Hence, by [18, Theorem 2],

$$
\int_{\mathbf{R}^{n}} W(x-y, t) d \mu^{+}(y) \leqslant \sum_{j=1}^{\infty} \mu^{+}\left(\left\{x_{j}\right\}\right) W\left(x-x_{j}, t\right),
$$

so that

$$
u(x, t)-\sum_{j=1}^{\infty} \mu^{+}\left(\left\{x_{j}\right\}\right) W\left(x-x_{j}, t\right) \leqslant-\int_{\mathbf{R}^{\prime \prime}} W(x-y, t) d \mu^{-}(y) \leqslant 0,
$$

and the result is proved.

Theorem 11 gives rise to another representation theorem, as follows. 
TheORem 12. Let $u=W \mu$ on $\left.\mathbf{R}^{n} \times\right] 0, c\left[\right.$, and let $C=\left\{x_{j}\right\}_{j \geqslant 1}$ be a sequence in $\mathbf{R}^{n}$. If there exist non-negative constants $A$ and $\boldsymbol{\kappa}$ such that

$$
\liminf _{t \rightarrow 0}|u(x, t)| \leqslant A \exp \left(\kappa\|x\|^{2}\right)
$$

m-a.e. on $\mathbf{R}^{n}$, and

$$
\liminf _{t \rightarrow 0}|u(x, t)|<\infty
$$

for all $x \in \mathbf{R}^{n} \backslash C$, then $u$ can be written in the form

$$
u(x, t)=h(x, t)+\sum_{j=1}^{\infty} \mu\left(\left\{x_{j}\right\}\right) W\left(x-x_{j}, t\right)
$$

on $\left.\mathbf{R}^{n} \times\right] 0, \min \left\{c,(4 \kappa)^{-1}\right\}\left[\right.$ if $\kappa>0$, on $\left.\mathbf{R}^{n} \times\right] 0, c[$ if $\kappa=0$, where $h$ is a temperature which satisfies

$$
|h| \leqslant A V_{\kappa} .
$$

PROOF. Applying Theorem 11 to $u$ we obtain

$$
u(x, t) \leqslant A V_{\kappa}(x, t)+\sum_{j=1}^{\infty} \mu^{+}\left(\left\{x_{j}\right\}\right) W\left(x-x_{j}, t\right),
$$

so that $u$ has a positive thermic majorant given by the expression on the right. This expression therefore majorizes the least positive thermic majorant of $u$, so that by [18, Theorem 2],

$$
\int_{\mathbf{R}^{n}} W(x-y, t) d \mu^{+}(y) \leqslant A V_{\kappa}(x, t)+\sum_{j=1}^{\infty} \mu^{+}\left(\left\{x_{j}\right\}\right) W\left(x-x_{j}, t\right) .
$$

Therefore

$$
0 \leqslant \int_{\mathbf{R}^{n}} W(x-y, t) d \mu^{+}(y)-\sum_{j=1}^{\infty} \mu^{+}\left(\left\{x_{j}\right\}\right) W\left(x-x_{j}, t\right) \leqslant A V_{\kappa}(x, t),
$$

and a similar argument applied to $-u$ gives

$$
0 \leqslant \int_{\mathbf{R}^{n}} W(x-y, t) d \mu^{-}(y)-\sum_{j=1}^{\infty} \mu^{-}\left(\left\{x_{j}\right\}\right) W\left(x-x_{j}, t\right) \leqslant A V_{\kappa}(x, t) .
$$

It follows that

$$
-A V_{\kappa}(x, t) \leqslant u(x, t)-\sum_{j=1}^{\infty} \mu\left(\left\{x_{j}\right\}\right) W\left(x-x_{j}, t\right) \leqslant A V_{\kappa}(x, t),
$$

which shows that $|h| \leqslant A V_{\kappa}$, as required.

There is a known representation theorem for a temperature $h$ which satisfies (25). For $n=1$, it is proved in [9, page 206]. Combining this with Theorem 12, we obtain a more explicit representation of $u$. 
COROLlary 1. If $u$ satisfies the hypotheses of Theorem 12, then there exists a function $f$ on $\mathbf{R}^{n}$ such that

$$
|f(x)| \leqslant A \exp \left(\kappa\|x\|^{2}\right)
$$

for all $x$, and

$$
u(x, t)=\int_{\mathbf{R}^{n}} W(x-y, t) f(y) d y+\sum_{j=1}^{\infty} \mu\left(\left\{x_{j}\right\}\right) W\left(x-x_{j}, t\right)
$$

on $\left.\mathbf{R}^{n} \times\right] 0, \min \left\{c,(4 \kappa)^{-1}\right\}\left[\right.$ if $\kappa>0$, on $\left.\mathbf{R}^{n} \times\right] 0, c[$ if $\kappa=0$.

Proof. By Theorem 12, $u$ has the representation (24). Define $f$ on $\mathbf{R}^{n}$ by

$$
f(x)=\limsup _{t \rightarrow 0} h(x, t) .
$$

Since $|h| \leqslant A V_{\kappa}$, it is obvious that $h$ has a positive thermic majorant $v$ such that

$$
\limsup _{t \rightarrow 0} v(x, t)<\infty
$$

for all $x$, and that $f(x)>-\infty$ for all $x$. The result now follows from [18, Theorem 1].

The special case of Theorem 12 in which $A=0$ and $\kappa=0$ gives us the following analogue of a theorem on harmonic functions on a disc in $\mathbf{R}^{2}$ due to Lohwater [12]. This corollary also contains, as the special case where $\mu$ is non-negative and $C$ is a singleton, a recent improvement [15, Theorem 5] of a theorem of Krzyżański [11, Theorem 5].

Corollary 2. Let $u=W \mu$ on $\left.\mathbf{R}^{n} \times\right] 0$, $c[$, let

$$
E=\left\{x \in \mathbf{R}^{n}: \lim _{t \rightarrow 0} u(x, t) \text { exists }\right\},
$$

and let $C=\left\{x_{j}\right\}_{j \geqslant 1}$ be a sequence of points in $E$. If $\lim _{t \rightarrow 0} u(x, t)=0$-a.e. on $E$, and $\lim _{t \rightarrow 0} u(x, t)$ is finite on $E \backslash C$, then

$$
u(x, t)=\sum_{j=1}^{\infty} \mu\left(\left\{x_{j}\right\}\right) W\left(x-x_{j}, t\right)
$$

for all $\left.(x, t) \in \mathbf{R}^{n} \times\right] 0, c[$.

Proof. By [5, Theorem 5.2], $m\left(\mathbf{R}^{n} \backslash E\right)=0$. It now follows that the hypotheses of Theorem 12 are satisfied, with $A=\kappa=0$, so that $u$ can be written in the form (24). Since $|h| \leqslant A V_{\kappa}=0$, the corollary is proved. 
Another consequence of Theorem 12 is roughly analogous to a result of Hall [8, Theorem 4] on holomorphic functions on a disc. His hypotheses allow approach to the boundary along arbitrary Jordan arcs, not just along radii, but require a uniform rate of growth where the values of the modulus are unbounded.

THEOREM 13. Let $u=W \mu$ on $\left.R^{n} \times\right] 0, c[$, and suppose that there are non-negative constants $A$ and $\kappa$ such that

(i) $\lim _{t \rightarrow 0}|u(x, t)| \leqslant A \exp \left(\kappa\|x\|^{2}\right) m$-a.e. on $\mathbf{R}^{n}$,

(ii) $\lim _{t \rightarrow 0}|u(x, t)|=\infty$ on a countable set $C$, and

(iii) $\lim _{t \rightarrow 0} t^{n / 2} u(x, t)=0$ for all $x \in C$.

Then $|u| \leqslant A V_{\kappa}$ on $\left.\mathbf{R}^{n} \times\right] 0, \min \left\{c,(4 \kappa)^{-1}\right\}\left[\right.$ if $\kappa>0$, on $\left.\mathbf{R}^{n} \times\right] 0, c[$ if $\kappa=0$, so that $u$ has a representation in the form

$$
u(x, t)=\int_{\mathbf{R}^{n}} W(x-y, t) f(y) d y
$$

for some function $f$ such that $|f(y)| \leqslant A \exp \left(\kappa\|y\|^{2}\right)$ for all $y$.

Proof. Hypotheses (i) and (ii), together with Theorem 12, imply that $u$ has the representation (24), where $\left\{x_{j}\right\}_{j \geqslant 1}=C$ and (25) holds. Using (iii) and (25), we obtain

$$
\lim _{t \rightarrow 0}\left(t^{n / 2} \sum_{j=1}^{\infty} \mu\left(\left\{x_{j}\right\}\right) W\left(x-x_{j}, t\right)\right)=\lim _{t \rightarrow 0} t^{n / 2}(u(x, t)-h(x, t))=0
$$

for all $x \in C$. For each non-negative integer $i$, we can write

$$
\sum \mu\left(\left\{x_{j}\right\}\right) W\left(x-x_{j}, t\right)=\int_{\mathbf{R}^{n}} W(x-y, t) d \nu_{i}(y),
$$

where the summation is taken over all $j \neq i$ and where, if $\delta_{j}$ denotes the Dirac $\delta$-measure concentrated at $x_{j}$,

$$
\nu_{i}=\sum \mu\left(\left\{x_{j}\right\}\right) \delta_{j} .
$$

Since $v_{i}\left(\left\{x_{j}\right\}\right)=0,[19$, Example 1] shows that

$$
t^{n / 2} \int_{\mathbf{R}^{n}} W(x-y, t) d v_{i}(y) \rightarrow 0
$$

as $t \rightarrow 0$. It therefore follows from (27) that

$$
\mu\left(\left\{x_{i}\right\}\right)=(4 \pi t)^{n / 2} \mu\left(\left\{x_{i}\right\}\right) W\left(x_{i}-x_{i}, t\right) \rightarrow 0 .
$$

Hence $\mu\left(\left\{x_{i}\right\}\right)=0$ for each $i$. It follows that $u=h$, and hence that $|u| \leqslant A V_{\kappa}$. The representation (26) now follows by an argument similar to the one used to prove Corollary 1 of Theorem 12. 


\section{References}

1. A. S. Besicovitch, 'A general form of the covering principle and relative differentiation of additive functions', Proc. Cambridge Philos. Soc. 41 (1945), 103-110.

2. A. S. Besicovitch, 'A general form of the covering principle and relative differentiation of additive functions. II', Proc. Cambridge Philos. Soc. 42 (1946), 1- 10.

3. M. Brelot, 'Remarques sur les zéros à la frontière des fonctions harmoniques positives', Boll. Un. Mat. Ital. 12 (1975), 314-319.

4. A. M. Bruckner, A. J. Lohwater and F. Ryan, 'Some non-negativity theorems for harmonic functions', Ann. Acad. Sci. Fenn. Ser. A. I. Math. Dissertationes 452 (1969).

5. J. L. Doob, 'Relative limit theorems in analysis', J. Analyse Math. 8 (1960/61), 289-306.

6. F. W. Gehring, 'The boundary behavior and uniqueness of solutions of the heat equation', Trans. Amer. Math. Soc. 94 (1960), 337-364.

7. R. Guenther, 'Representation theor ems for linear second-order parabolic partial differential equations', J. Math. Anal. Appl. 17 (1967), 488-501.

8. R. L. Hall, 'On the asymptotic behaviour of functions holomorphic in the unit disc', Math. $Z$. 107 (1968), 357-362.

9. I. I. Hirschman and D. V. Widder, The convolution transform (Princeton University Press, Princeton, N. J., 1955).

10. Ü. Kuran, 'Some extension theorems for harmonic, superharmonic and holomorphic functions', J. London Math. Soc. 22 (1980), 269-284.

11. M. Krzyżański, 'Sur les solutions non négatives de l'équation linéaire normale parabolique', Rev. Roumaine Math. Pures Appl. 9 (1964), 393-408.

12. A. J. Lohwater, 'A uniqueness theorem for a class of harmonic functions', Proc. Amer. Math. Soc. 3 (1952), 278-279.

13. P. C. Rosenbloom, 'Linear equations of parabolic type with constant coefficients', Contributions to the theory of partial differential equations (Princeton University Press, Princeton, N. J., 1954, 191-200).

14. N. A. Watson, 'Classes of subtemperatures on infinite strips', Proc. London Math. Soc. 27 (1973), 723-746.

15. N. A. Watson, 'Differentiation of measures and initial values of temperatures', J. London Math. Soc. 16 (1977), 271-282.

16. N. A. Watson, 'Thermal capacity', Proc. London Math. Soc. 37 (1978), 342-362.

17. N. A. Watson, 'Uniqueness and representation theorems for the inhomogeneous heat equation', J. Math. Anal. Appl. 67 (1979), 513-524.

18. N. A. Watson, 'Positive thermic majorization of temperatures on infinite strips', J. Math. Anal. Appl. 68 (1979), 477-487.

19. N. A. Watson, 'Initial singularities of Gauss-Weierstrass integrals and their relations to Laplace transforms and Hausdorff measures', J. London Math. Soc. 21 (1980), 336-350.

\section{Department of Mathematics \\ University of Canterbury \\ Christchurch \\ New Zealand}

